\title{
Intradural Extramedullary Ependymoma at Lumbar (L1-L4 Level) Spine: A Suspicious Case and Literature Review
}

\author{
Akbar Shoukat Ali*, Muhammad Sameer Qureshi, Javed Ahmed, Gomand Beekho Sonekhi and Attaullah Ahmed \\ Institute of Nursing, Dow University of Health Sciences, Pakistan
}

\begin{abstract}
Ependymomas constitute $4-6 \%$ of primary central nervous system tumors. Spinal ependymomas are most frequently found in intramedullary region but few cases of intradural extramedullary ependymoma have also been reported. We report a 24-year-old male patient with a suspected case of intradural extramedullary ependymoma. Magnetic resonance images of the lumbar spine depicted an intradural mass from L1-L4 level. The spinal lesion was isointense on T1-weighted images and hyperintense on T2-weighted images, relative to the spinal cord. Laminectomy L1-L4 with gross-total excision was performed. Histopathological examination was inconclusive but suggested the possibility of ependymoma. Neurological recovery was initially observed but after few months symptoms worsened.
\end{abstract}

Keywords: Suspicious; Ependymoma; Intramedullary; Extra medullary

\section{Introduction}

Tumors of spinal cord constitute $15 \%$ of Central Nervous System (CNS) tumors $[1,2]$ They can be categorized as intradural or extradural, the former being either intramedullary (involving the substance of spinal cord) or extramedullary (outside the spinal cord) depending on their location. Ependymomas are the most frequent glial cells derived tumors found in the spinal cord. Classically, spinal ependymomas are intradural intramedullary tumors with predominance in adults. Intradural extramedullary spinal ependymomas are rare. Current literature suggests that very few cases of such tumors have been reported (Table 1). Although these spinal tumors are rare and benign but compressive lesions secondary to ependymoma could lead to range of symptoms from lumbago (lower back pain), sensory and motor disturbances to acute paraplegia [3-7]. Herein, we report a rare and suspected case of intradural extramedullary ependymoma in a 24 -year-old male.

\section{Case Report}

A 24-year-old male presented with history of mid/lower lumbago (back pain) for 1 month, progressive weakness of lower limbs for the last 5-6 days, and fecal and urinary retention for the last 3 days. Past medical history was unremarkable for trauma. On comprehensive neurological assessment, there was decreased muscle tone in both lower limbs, with overall grade- 2 and grade- 3 power in left and right lower limb muscle groups, respectively. Deep tendon reflexes (DTR) were absent in all four limbs. Other spine examinations were inconclusive.

\section{MRI screening}

Detailed MRI screening suggested evidence of a large abnormal lesion within spinal canal starting at the level of L1 vertebra and extending down to the lower border of L4 vertebral body (Figure 1a). The lesion appeared isointense to cord on T1-weighted image, while hyper intense on T2-weighted image. MRI features were consistent with neoplastic lesion, likely of nerve sheath origin. Intradural extramedullary tumor was suspected as the initial diagnosis.

\section{Surgery}

Laminectomy L1-L4, durotomy and gross-total excision of spinal mass was performed under general anesthesia. Midline spinal incision was given from L1-L4 in order to remove the mass. Intra-operative findings were multiple irregular gray brown soft bodies collectively measuring $3 \times 2.8 \times 0.5 \mathrm{~cm}$ with hemorrhage. Piecemeal excision was carried out with Redivac drain placement. No post-operative complications were observed.

\section{Histopathology}

Histopathological examination showed rounded nuclei with eosinophilic cytoplasm focally showing nesting pattern with interspersed thick-walled vessels. At places, neoplastic cells were arranged around vessels. A panel of immunohistochemical examination was performed for antibodies against CD99, GFAP, CKAE1/AE3, S100, Dermis, CD138. Immunohistochemical staining was negative for all except CD99. Final histology report demonstrated inconclusive result but also suggested that the possibility of ependymoma could not be entirely excluded.

\section{Discussion}

Spinal cord tumors tumors account for $15 \%$ of all CNS tumors.1, 2 Most prevalent location of such tumors is found to be intradural intramedullary though cases of intradural extramedullary ependymoam have also been reported in literature along with this case [3] Intradural extramedullary ependymomas are more prevalent among females and in 5th decade of life.6 Hormonal factor had been indicted as the major reason for female predominance by Duffau et al. in their review paper; however, its definite involvement is not well appreciated by other studies [8]. Contrary to most of the previous case reports, our patient was male with age range almost similar to that reported by Iunes et al.; 24-69 years and 23-87 years in our review of literature (2000-2013), respectively [3,4,6-19].

Magnetic Resonance Imaging (MRI) was the choice of neuroimaging modality since it can well localize the lesion $[7,8]$. Thoracic spine has been found to be the most frequent location of intradural extramedullary ependymoma $[3,4,6-9,11,12,16-19]$. Compared with earlier cases, our case was among the few with the lumbar spine involvement (suggestive

${ }^{*}$ Corresponding author: Akbar Shoukat Ali, Institute of Nursing, Dow University of Health Sciences, Pakistan,Tel: +93-312-8624328; E-mail: akber.shoukat.ali@gmail.com

Received July 16, 2015; Accepted August 13, 2015; Published August 20, 2015

Citation: Ali AS, Qureshi MS, Ahmed J, Sonekhi GB, Ahmed A (2015) Intradural Extramedullary Ependymoma at Lumbar (L1-L4 Level) Spine: A Suspicious Case and Literature Review. J Clin Case Rep S3: 004. doi:10.4172/2165-7920.S3-004

Copyright: ( 2015 Ali AS, et al. This is an open-access article distributed under the terms of the Creative Commons Attribution License, which permits unrestricted use, distribution, and reproduction in any medium, provided the original author and source are credited. 
Citation: Ali AS, Qureshi MS, Ahmed J, Sonekhi GB, Ahmed A (2015) Intradural Extramedullary Ependymoma at Lumbar (L1-L4 Level) Spine: A Suspicious Case and Literature Review. J Clin Case Rep S3: 004. doi:10.4172/2165-7920.S3-004

Page 2 of 4

\begin{tabular}{|c|c|c|c|c|c|c|c|c|c|}
\hline Author & Age & Gender & Location & Symptoms & $\begin{array}{l}\text { Preoperative } \\
\text { diagnosis }\end{array}$ & $\begin{array}{l}\text { Histologic } \\
\text { diagnosis }\end{array}$ & Prognosis & F/U Period & Recurrence \\
\hline Duffau et al. [8] & 43 & Female & Thoracic (T1-T8) & $\begin{array}{c}\text { Paraplegia, sensory } \\
\text { abnormality, bladder } \\
\text { dysfunction }\end{array}$ & Not mentioned & $\begin{array}{c}\text { Benign } \\
\text { Ependymoma }\end{array}$ & $\begin{array}{l}\text { Good neurological } \\
\text { recovery }\end{array}$ & 24 months & No recurrence \\
\hline $\begin{array}{c}\text { Bavbek et al. } \\
\text { [10] }\end{array}$ & 46 & Male & Lumbar (L1-L2) & $\begin{array}{l}\text { Monoparesthesia, } \\
\text { urinary, fecal } \\
\text { incontinence }\end{array}$ & Neurofibroma & $\begin{array}{l}\text { Myxopapillary } \\
\text { ependymoma }\end{array}$ & $\begin{array}{l}\text { Neurological } \\
\text { improvement }\end{array}$ & 6 weeks & No recurrence \\
\hline $\begin{array}{c}\text { Robles et al. } \\
{[11]}\end{array}$ & 47 & Female & D2-D3 & Not mentioned & $\begin{array}{l}\text { Neurinoma, } \\
\text { meningioma }\end{array}$ & $\begin{array}{l}\text { Benign classic } \\
\text { ependyoma }\end{array}$ & $\begin{array}{c}\text { No complications } \\
\text { were seen, good } \\
\text { neurological recovery }\end{array}$ & 1 year & $\begin{array}{l}\text { Recurrence } \\
\text { with anaplastic } \\
\text { transformation }\end{array}$ \\
\hline Graca [12] & 67 & Female & $\begin{array}{c}\text { Thoracic (T5-T6 } \\
\text { to T8) }\end{array}$ & $\begin{array}{c}\text { Sensory abnormality, } \\
\text { GD }\end{array}$ & $\begin{array}{l}\text { Arachnoid cyst } \\
\text { with spinal cord } \\
\text { compression }\end{array}$ & $\begin{array}{l}\text { WHO grade II } \\
\text { ependymoma }\end{array}$ & $\begin{array}{l}\text { Worsening of } \\
\text { symptoms }\end{array}$ & 3 months & $\begin{array}{l}\text { Recurrent cystic } \\
\text { lesion }\end{array}$ \\
\hline $\begin{array}{l}\text { Schuurmans et } \\
\text { al. [13] }\end{array}$ & 29 & Female & Cervical (C3-C6) & $\begin{array}{l}\text { Neck pain, muscular } \\
\text { weakness, urinary } \\
\text { dysfunction }\end{array}$ & Not mentioned & $\begin{array}{l}\text { WHO grade } \\
\text { III anaplastic } \\
\text { ependymoma }\end{array}$ & $\begin{array}{l}\text { Neurological } \\
\text { improvement }\end{array}$ & 2 years & $\begin{array}{l}\text { Intracranial } \\
\text { extracerebral } \\
\text { metastasis }\end{array}$ \\
\hline $\begin{array}{l}\text { Bonfield et al. } \\
\qquad[14]\end{array}$ & 87 & Female & Lumbar (L3) & $\begin{array}{l}\text { Hip, thigh pain, } \\
\text { bladder dysfunction }\end{array}$ & Not mentioned & $\begin{array}{l}\text { Extramedullary } \\
\text { ependymoma } \\
\text { near conus } \\
\text { medullaris }\end{array}$ & $\begin{array}{c}\text { Postoperative course } \\
\text { was uneventful }\end{array}$ & $\begin{array}{c}\text { Not } \\
\text { mentioned }\end{array}$ & Not mentioned \\
\hline Guppy et al. [4] & 50 & Male & Thoracic (T5-T6) & $\begin{array}{c}\text { Progressive } \\
\text { weakness, sensory } \\
\text { abnormality }\end{array}$ & Not mentioned & $\begin{array}{l}\text { WHO grade } \\
\text { III anaplastic } \\
\text { ependymoma }\end{array}$ & $\begin{array}{l}\text { Neurological } \\
\text { improvement } \\
\text { observed }\end{array}$ & 6 months & No recurrence \\
\hline lunes et al. [9] & 32 & Male & $\begin{array}{c}\text { Bulbomedullary } \\
\text { juntion, cervical } \\
\text { (C2-C5), thoracic } \\
\text { (T5-T11), Lumbar } \\
\text { (L2, L4-L5), and } \\
\text { Sacrum }\end{array}$ & $\begin{array}{l}\text { Lower limb } \\
\text { paresthesia, GD, } \\
\text { urinary retention }\end{array}$ & Not mentioned & $\begin{array}{l}\text { WHO garde II } \\
\text { ependymoma }\end{array}$ & $\begin{array}{l}\text { Tumor Progression } \\
\text { and death }\end{array}$ & 10 months & $\begin{array}{l}\text { Tumor } \\
\text { recurrence }\end{array}$ \\
\hline Son et al. [15] & 57 & Female & Cervical (C2-C6) & $\begin{array}{c}\text { Neck pain, muscular } \\
\text { weakness }\end{array}$ & $\begin{array}{l}\text { Neurinoma, } \\
\text { neurofibroma or } \\
\text { meningioma }\end{array}$ & $\begin{array}{l}\text { WHO grade II } \\
\text { ependymoma }\end{array}$ & $\begin{array}{l}\text { Neurologic } \\
\text { improvement }\end{array}$ & 5 years & No recurrence \\
\hline \multirow{2}{*}{ Landriel et al. [6] } & 30 & Male & D2 and D12-L1 & $\begin{array}{c}\text { LBP, urinary } \\
\text { disturbance, GD, } \\
\text { sensory abnormality }\end{array}$ & Not mentioned & $\begin{array}{l}\text { WHO grade I } \\
\text { Myxopapillary } \\
\text { ependymoma }\end{array}$ & $\begin{array}{l}\text { Lower limb paresis } \\
\text { and radicular pain } \\
\text { improved }\end{array}$ & 10 years & No recurrence \\
\hline & 32 & Male & D10 & $\begin{array}{l}\text { LBP, sensory } \\
\text { abnormality }\end{array}$ & Not mentioned & $\begin{array}{l}\text { WHO grade I } \\
\text { Myxopapillary } \\
\text { ependymoma }\end{array}$ & $\begin{array}{l}\text { No improvement in } \\
\text { referred symptoms }\end{array}$ & 1 year & No recurrence \\
\hline Ha et al. [7] & 36 & Female & $\begin{array}{l}\text { Cervical (C6)- } \\
\text { Thoracic(T4) }\end{array}$ & Pain, paraplegia & Not mentioned & $\begin{array}{l}\text { WHO garde II } \\
\text { ependymoma }\end{array}$ & $\begin{array}{l}\text { Neurological } \\
\text { improvement }\end{array}$ & 6 months & No recurrence \\
\hline $\begin{array}{c}\text { Gardener et al. } \\
{[16]}\end{array}$ & 27 & Female & Thoracic (T2-T7) & $\begin{array}{l}\text { Band-like sensation } \\
\text { in chest and urinary } \\
\text { symptoms }\end{array}$ & Not mentioned & Ependymoma & $\begin{array}{l}\text { Neurological } \\
\text { improvement }\end{array}$ & 8 months & No recurrence \\
\hline Kim et al. [17] & 48 & Female & Thoracic (T7-T9) & $\begin{array}{l}\text { Radiating pain, motor } \\
\text { disturbance, urinary } \\
\text { incontinence }\end{array}$ & Not mentioned & $\begin{array}{l}\text { WHO grade } \\
\text { III anaplastic } \\
\text { ependymoma }\end{array}$ & $\begin{array}{l}\text { Neurological } \\
\text { deterioration }\end{array}$ & 14 months & $\begin{array}{c}\text { Newly developed } \\
\text { mass at } \\
\text { lumbosacral } \\
\text { region }\end{array}$ \\
\hline $\begin{array}{l}\text { Moriwaki et } \\
\text { al. [3] }\end{array}$ & 23 & Female & Thoracic (T4-T6) & $\begin{array}{l}\text { Pain, sensory and } \\
\text { motor disturbances, } \\
\text { GD }\end{array}$ & $\begin{array}{l}\text { Schwannoma or } \\
\text { Meningioma }\end{array}$ & $\begin{array}{l}\text { WHO grade II } \\
\text { ependymoma }\end{array}$ & $\begin{array}{l}\text { Neurologic } \\
\text { improvement with } \\
\text { mild paresthesia } \\
\text { and pain in the left } \\
\text { abdominal region }\end{array}$ & 1.5 years & $\begin{array}{l}\text { Recurring mass } \\
\text { at T4-T5 level }\end{array}$ \\
\hline $\begin{array}{l}\text { Perez-Bovet et } \\
\quad \text { al. [18] }\end{array}$ & 36 & Female & Multiple locations & $\begin{array}{l}\text { Headache, CNP, } \\
\text { Hemiparesis }\end{array}$ & Not mentioned & $\begin{array}{l}\text { WHO grade } \\
\text { III anaplastic } \\
\text { ependymoma }\end{array}$ & $\begin{array}{c}\text { No neurological } \\
\text { recovery, patient } \\
\text { died after } 7 \text { weeks of } \\
\text { diagnosis }\end{array}$ & $\begin{array}{c}\text { Not } \\
\text { mentioned }\end{array}$ & Not mentioned \\
\hline $\begin{array}{c}\text { Samanci et al. } \\
{[19]}\end{array}$ & 34 & Male & $\begin{array}{l}\text { Thoracic (T7) to } \\
\text { Lumbar (L2) }\end{array}$ & $\begin{array}{c}\text { Backache, } \\
\text { weakness, bladder } \\
\text { disturbance }\end{array}$ & Not mentioned & $\begin{array}{l}\text { Myxopapillary } \\
\text { ependymoma }\end{array}$ & $\begin{array}{l}\text { Neurological } \\
\text { improvement }\end{array}$ & 3 years & Not mentioned \\
\hline Present Case & 24 & Male & Lumbar (L1-L4) & $\begin{array}{l}\text { Backache, lower limb } \\
\text { weakness, fecal and } \\
\text { urinary retention }\end{array}$ & $\begin{array}{l}\text { Intradural } \\
\text { extramedullary } \\
\text { tumor }\end{array}$ & $\begin{array}{c}\text { Possible } \\
\text { Ependymoma }\end{array}$ & $\begin{array}{l}\text { Neurological } \\
\text { Recovery }\end{array}$ & $\begin{array}{c}\text { Not } \\
\text { mentioned }\end{array}$ & Not mentioned \\
\hline
\end{tabular}

GD: Gait disturbance; LBP: Low Back Pain; CNP: Cranial Nerve Palsy

Table 1: Literature review on Intradural Extramedullary Ependymoma.

of conus ependymoam) (Figure $1 \mathrm{~b}$ and 1c), [9,10,14,18,19]. Tumor location directly correlates with the symptomatology.4 Pain, sensory and motor deficits and bladder dysfunction were the most commonly reported symptoms in previously published cases $[3,4,6-10,12-17,19]$. Similar clinical features were also found in our case.
Initial neuroimaging findings are usually non-specific in terms of firm diagnosis.15 MRI findings in our case were consistent with those of previous cases; the tumor appeared isointense to spinal cord on T1-weighted images, while hyperintense on T2-weighted images $[4,6,9,12,15]$. Our literature review depicted that initial diagnosis 
Citation: Ali AS, Qureshi MS, Ahmed J, Sonekhi GB, Ahmed A (2015) Intradural Extramedullary Ependymoma at Lumbar (L1-L4 Level) Spine: A Suspicious Case and Literature Review. J Clin Case Rep S3: 004. doi:10.4172/2165-7920.S3-004
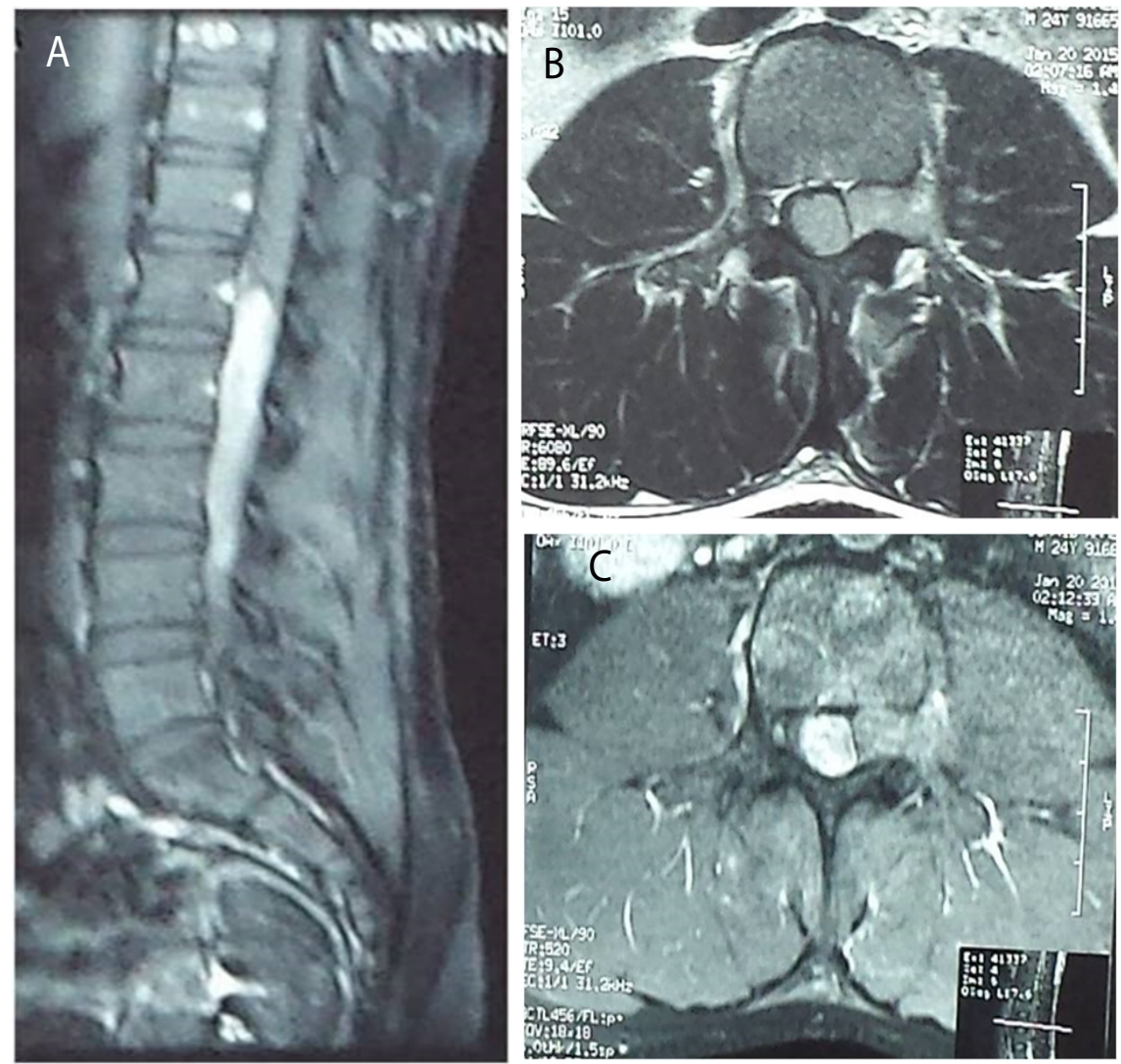

Figure 1: (a) MRI Image of the Lumbar Spine illustrating Intradural Extramedullary Lesion in the Lumbar Region, L1-L4. (b and c): MRI Images of the Lumbar Spine illustrating Intradural Extramedullary Lesion in the Lumbar Region, L1-L4.

was meningioma, neurinoma, neurofibroma, or schwannoma $[3,10,11,15]$. In the present case, pre-operative diagnosis was intradural extramedullary tumor.

Histopathologically, the case was suspicious which is striking and makes this case new in this entity. Despite extensive clinical and histopathological work up, the diagnosis remained elusive. The case is first of its kind in history with ambiguous histology result. On one side, MRI reports are clearly suggestive of conus ependymoma while on the other hand histology results are inconclusive but still suspection of ependymoma is open.

Surgically, gross total resection of the tumor has been regarded as the best approach for good prognosis as was done in the current case. Sonneland et al. also mentioned good survival results for patients who underwent gross total resection compared to those with partial resection of the tumor.20 Intradural extramedullary ependymomas have been described as benign tumors in the literature but countable cases have followed malignant sequelae $[3,4,11,13,17,18]$. No adjunctive radiotherapy was given to our patient since there was no evidence of residual tumor or any malignant transformation. Post-operative radiotherapy should be warranted in case of malignant transformation.7 Keeping in mind the possibility of malignant sequelae and recurrence, [3,4,9,11-13,17,18]. patient was guided for regular follow-up but no follow up was seen after few months.

\section{Conclusion}

In toto, intradural extramedullary ependymomas are very rare in this part of the world. The present case of intradural extramedullary tumor is unique and should be subjected to scrutinize to identify what is going on at the molecular level.

\section{References}

1. Van Goethem JW, van den Hauwe L, Ozsarlak O, De Schepper AM, Parizel PM (2004) Spinal tumors. Eur J Radiol 50: 159-176.

2. Sonawane DV, Jagtap SA, Mathesul AA (2012) Intradural extramedullary capillary hemangioma of lower thoracic spinal cord. Indian J Orthop 46: 475478.

3. Moriwaki T, Iwatsuki K, Ohnishi Y, Umegaki M, Ishihara M, et al. (2013) Intradural Extramedullary Spinal Ependymoma: A Case Report of Malignant Transformation Occurring. Asian Spine J 7: 139-142.

4. Guppy KH, Hou L, Moes GS, Sahrakar K (2011) Spinal intradural, extramedullary anaplastic ependymoma with an extradural component: Case report and review of the literature. Surg Neurol Int 2: 119.

5. Pan E, Prados MD (2003) Spinal Cord Tumors Holland-Frei Cancer Medicine. (6th edn), Hamilton (ON): BC Decker, Holland.

6. Landriel F, Ajler P, Tedesco N, Bendersky D, Vecchi E (2012) Multicentric extramedullary ependymomas: Two case reports and literature review. Surg Neurol Int 3:102.

7. Ha SM, Shin DA (2012) Intradural Extramedullary Ependymoma with Spinal Root Attachment. Korean J Spine 9: 250-252.

8. Duffau H, Gazzaz M, Kujas M, Fohanno D (2000) Primary intradural extramedullary ependymoma: Case report and review of the literature. Spine 25: 1993-1995.

9. Iunes EA, Stavale JN, Ansai R, Onishi FJ, de Paiva Neto MA, et al. (2011) Multifocal intradural extramedullary ependymoma: Case report. J Neurosurg Spine 14: 65-70. 
Citation: Ali AS, Qureshi MS, Ahmed J, Sonekhi GB, Ahmed A (2015) Intradural Extramedullary Ependymoma at Lumbar (L1-L4 Level) Spine: A Suspicious Case and Literature Review. J Clin Case Rep S3: 004. doi:10.4172/2165-7920.S3-004

10. Bavbek M, Altinors MN, Caner HN, Bilezikci B, Agildere M (2001) Lumbar myxopapillary ependymoma mimicking neurofibroma. Spinal Cord 39: 449-452.

11. Robles SG, Saldana C, Boto GR, Martinez A, Zamarron AP et al. (2005) Intradural extramedullary spinal ependymoma: A benign pathology? Spine 30: E251-254.

12. Graca J, Gultasli N, D'Haene N, Brotchi J, Salmon I et al. (2006) Cystic extramedullary ependymoma. AJNR Am J Neuroradiol 27: 818-821.

13. Schuurmans M, Vanneste JA, Verstegen MJ, Van Furth WR (2006) Spinal extramedullary anaplastic ependymoma with spinal and intracranial metastases. J Neurooncol 79: 57-59.

14. Bonfield CM, Amin D, Hamilton RL, Gerszten PC (2011) Extramedullary ependymoma near the conus medullaris with lumbar nerve root attachment: Case report. Neurosurgery 68: E831-834.
15. Son DW, Song GS, Han IH, Choi BK (2011) Primary extramedullary ependymoma of the cervical spine: Case report and review of the literature. $J$ Korean Neurosurg Soc 50: 57-59.

16. Gardener L, Kasliwal MK, Hempeck N, Utset M, Gandhi YN (2013) Ependymoma: Unusual differential for totally extramedullary intraspinal tumor. Neurol India 61 : 687-690.

17. Kim SY, Kim SW (2006) Primary intradural extramedullary myxopapillary ependymoma. J Korean Neurosurg Soc 39: 382-384.

18. Perez-Bovet J, Rimbau-Munoz J, Martin-Ferrer S (2013) Anaplastic ependymoma with holocordal and intracranial meningeal carcinomatosis and holospinal bone metastases. Neurosurgery 72: E497-503.

19. Samanci Y, Celik SE (2013) Extramedullary Myxopapillary Ependymoma of the Filum Terminale Associated With Syringomyelia: A Case Report. Romanian Neurosurgery 4: 393-396.

This article was originally published in a special issue, Neurology and Neuropsychiatry Case Reports handled by Editor(s). Dr. Angelo Lavano, Magna Grcecia University, Italy. 